\title{
Acute Type A Aortic Dissection with Iliac Malperfusion in a Patient with Scimitar Syndrome
}

\author{
Jae Hang Lee, ${ }^{1}$ MD, Jin-Ho Choi, ${ }^{1}$ MD and Eung-Joong Kim, ${ }^{1}$ MD
}

\begin{abstract}
Summary
We encountered a 35-year-old male patient with scimitar syndrome, acute type A aortic dissection, and severe iliac malperfusion. He presented with pain and weakness of the left lower extremity and underwent ascending aorta replacement through sternotomy and right anterior thoracotomy. Left iliac artery flow was recovered after the operation and the patient was discharged on the tenth day without any complication. The purpose of this report is to discuss the considerations in aortic surgery in patients with scimitar syndrome, and the surgical principles for treatment of aortic dissection with critical limb ischemia.
\end{abstract}

(Int Heart J 2018; 59: 220-222)

Key words: Aorta, Partial anomalous pulmonary venous return

$\mathrm{S}$ cimitar syndrome is characterized by anomalous drainage of the right pulmonary veins, hypoplasia of the right lung, dextroposition of the heart, and anomalous systemic arterial supply to the right lung. This is a very rare complex anomaly that occurs in approximately two people per 100,000 population, ${ }^{1)}$ and there is no published case of acute type A aortic dissection in a patient with scimitar syndrome.

Aortic dissection is a life-threatening condition, and malperfusion syndrome occurs when there is an insufficient blood supply to the vital organs due to the dissection. If end-organ ischemia persists, infarct-induced malfunction will occur, and delayed treatment will have a negative impact on patient outcome due to reperfusion injury. ${ }^{2.3)}$ Malperfusion is a common complication of acute or chronic aortic dissection, but complete occlusion due to long-segment thrombus in the false lumen is a rare finding.

\section{Case Report}

A 35-year-old man visited a local hospital with a cold and painful left leg. Type A aortic dissection was found, and he was transferred to our hospital. He had worsening back pain and chest pain at the time of admission to our emergency department. His upper extremity blood pressure was $150 / 80 \mathrm{mmHg}$ and the pulse rate was stable at 70 per minute, but the lower extremity blood pressure was not measured.

A chest X-ray showed dextroposition of the heart, and electrocardiogram (ECG) confirmed normal sinus rhythm (Figure 1). The results of laboratory data were generally good, except leukocytosis (white blood cell count was 15,000). We did not check muscle enzymes, such as creatine kinase or myoglobin. Computed tomographic angiogram (CTA) confirmed type A (DeBakey type I) acute aortic dissection and hypoplasia of the right lung (Figure 2). There was a partial anomalous pulmonary venous connection (PAPVC), in which the left upper pulmonary vein drained into the innominate vein, and the right lower pulmonary vein drained into the IVC. There was systemic arterial supply to the right lower lobar posterior basal segment with right middle lobar pulmonary arterial hypogenesis, which can be associated with scimitar syndrome. The left common iliac artery was completely occluded by a long-segment false lumen thrombosis extending $10 \mathrm{~cm}$ from the entrance, with arterial flow from the distal external iliac artery.

We performed emergent repair of the aortic dissection via median sternotomy and right anterior thoracotomy with the patient in the right semilateral position. Arterial cannulation was performed with a $10 \mathrm{~mm}$ Dacron graft connected to the right subclavian artery without separate incision. SVC cannulation was performed through the right atrial appendage, and IVC cannulation was performed via the femoral vein. A vent cannula was inserted through left atrial appendage (Figure 3). Cardioplegic solution was not injected until the patient's body temperature was lowered to $22^{\circ} \mathrm{C}$, and during rewarming, direct cardioplegia injection was performed through the coronary orifice. There was a $2 \mathrm{~cm}$ horizontal intimal tear $1 \mathrm{~cm}$ above the left coronary orifice, and the main operation was replacement of the ascending aorta with a $30 \mathrm{~mm} \mathrm{Da}-$ cron graft. Cardiopulmonary bypass time was 213 minutes and selective antegrade cerebral perfusion time was 32 minutes.

From the ${ }^{1}$ Department of Thoracic and Cardiovascular Surgery, Dongguk University Ilsan Hospital, Goyang, Gyeonggi, Korea.

Address for correspondence: Jae Hang Lee, MD, Department of Thoracic and Cardiovascular Surgery, Dongguk University Ilsan Hospital, Siksa-dong, Ilsandong-gu, Goyang-si, Gyeonggi-do, 410-773, Korea. E-mail: truemed@hotmail.com

Received for publication January 25, 2017. Revised and accepted March 22, 2017.

Released in advance online on J-STAGE December 27, 2017.

doi: 10.1536/ihj.17-051

All rights reserved by the International Heart Journal Association. 

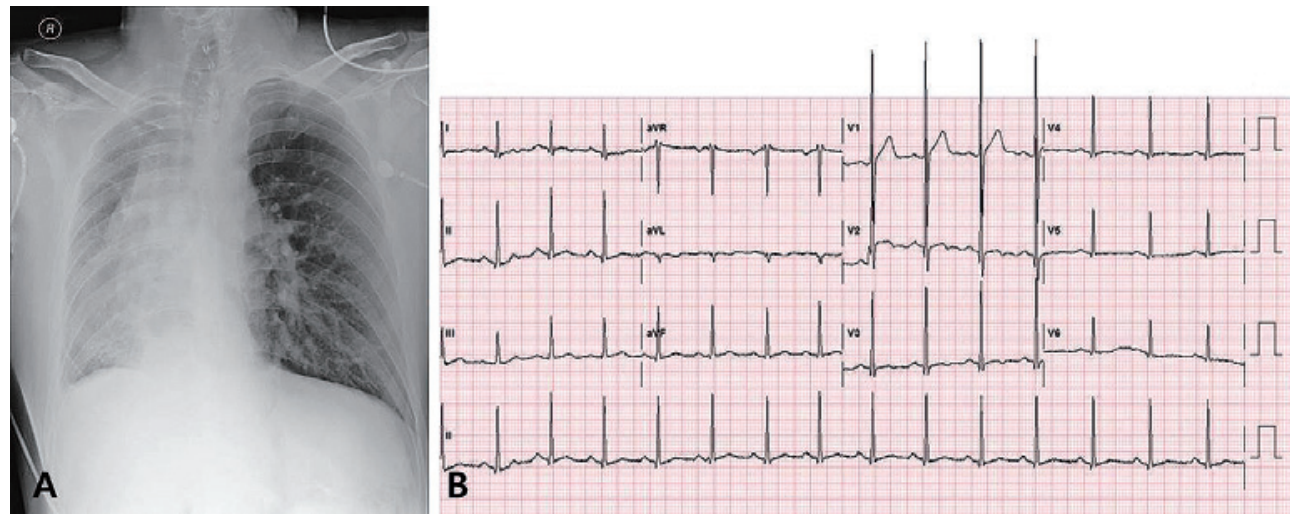

Figure 1. Preoperative chest $\mathrm{X}$-ray (A) and electrocardiogram (B).

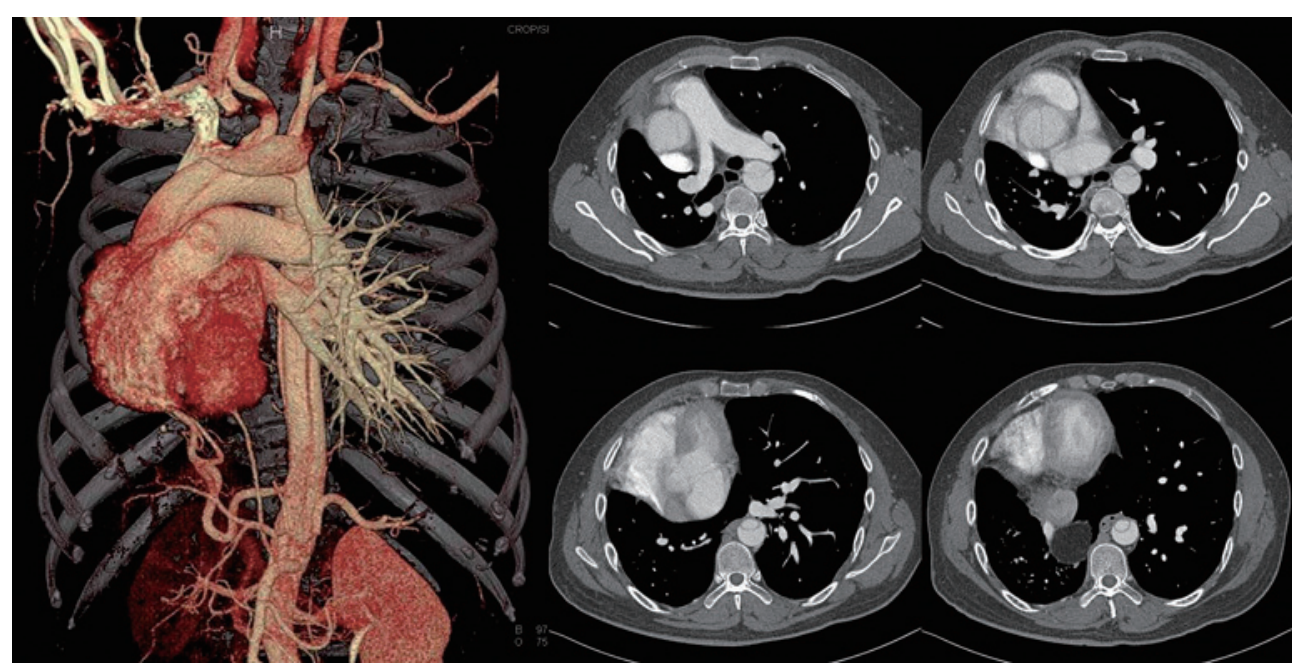

Figure 2. Computed tomographic angiography showing the deviated heart and hypoplasia of the right lung.

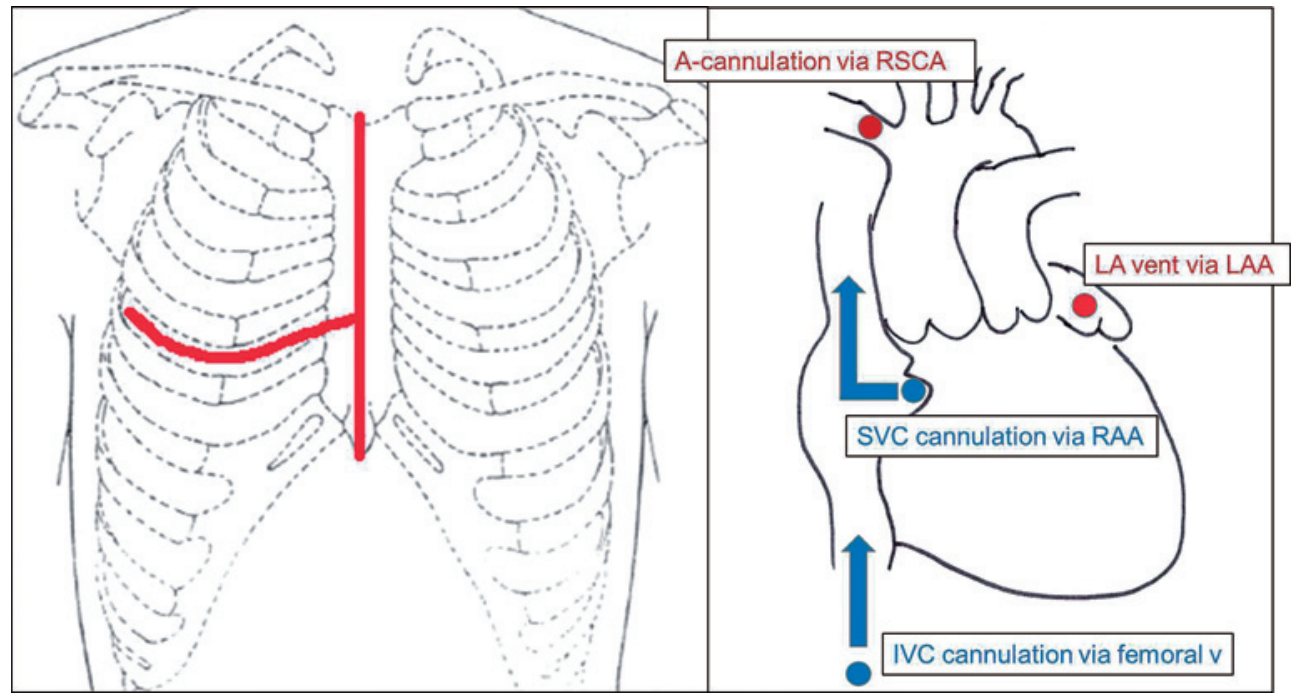

Figure 3. Incision and cannulation sites. 
The patient was extubated at 6 hours after surgery, and the left leg pain decreased from 12 hours after surgery. The left lower extremity blood pressure was not checked on the day of surgery, but the initial anklebrachial index (ABI) of 0.60 had improved to 0.74 by the day before discharge, and improved left iliac flow was confirmed at pre-discharge CTA. At postoperative echocardiography, there was no atrial septal defect, left ventricular function was good, and there were no other findings besides anomalies observed on CTA. The pathology examination showed medial aortic degeneration. The patient was transferred to the general ward 2 days postoperatively and was discharged 10 days postoperatively. He has remained under postoperative outpatient follow-up for 6 months without any complications.

\section{Discussion}

Scimitar syndrome was first described by Halasz, et $a l{ }^{4)}$ The name is based on the observation that the shape of the abnormal right pulmonary vein draining into the IVC on chest X-ray looks like a scimitar. Most patients with scimitar syndrome have mild symptoms and do not have an indication for surgery because they do not have large L-R shunts. However, surgical treatment may be indicated for patients with large L-R shunts or for those with other associated cardiac anomalies. There are many considerations for open-heart surgery in patients with scimitar syndrome; foremost among them are the patient's position, the incision sites, and the cannulation sites.

Because of the dextroposition of the heart, it is often difficult to perform surgery through median sternotomy alone. Therefore, we placed our patient in a right semilateral position with a right anterior thoracotomy in mind. This position is commonly used in robotic surgery, and right anterior thoracotomy is recommended because it is difficult to obtain an adequate visual field around the right atrium and IVC with median sternotomy alone.

Cannulation is more likely to be a problem in patients with scimitar syndrome. Generally, during surgery for type A aortic dissection, axillary cannulation is performed by a separate incision. However, in this patient, arterial cannulation was possible without additional incision because the right subclavian artery was visible in the sternotomy line. The right atrium is relatively small and collapsed, and bicaval cannulation is recommended rather than single venous cannulation. However, because obtaining a visual field for this is also difficult, it can be useful to perform IVC cannulation via the femoral vein. Although venting of the left heart is generally done through the right upper pulmonary vein, in this patient, it was difficult to identify the pulmonary vessels, whereas the rightward deviation of his heart allowed sufficient visibility of the left atrial auricle, and it was preferable to insert the vent catheter through here.

Persistent left IVC is common in scimitar syndrome. This point is also very important for open-heart surgery because myocardial protection may not be sufficient if the left IVC is overlooked during retrograde cardioplegia. We routinely wait until the lowest body temperature is reached before injecting a cardioplegic solution during ascending aorta replacement, and direct antegrade cardioplegia is performed while rewarming.

It is difficult to decide the extent of the surgery in the emergent situation. We performed ascending aortic replacement only because the patient has been doing well without cyanosis. So we think that left to right shunt through the scimitar vein is minimal in this patient, and we decided not to perform the repair of abnormal pulmonary venous returns.

The complex of acute type A aortic dissection and scimitar syndrome is very rare, and there are no literatures about that. There are only some case reports about the complex of PAPVC and type A aortic dissection, or scimitar syndrome and type B aortic dissection., ${ }^{5,6}$ We expect this report to be the first case of acute type A aortic dissection with scimitar syndrome.

In conclusion, in patients with scimitar syndrome who are undergoing open-heart surgery, right anterior thoracotomy is a safe option for securing a good field of vision. In these patients, cannulation at the standard sites is often difficult, and alternative sites should be chosen carefully. Finally, in cases of type A aortic dissection accompanied by iliac malperfusion, even with long-segment thrombotic occlusion, it is ideal to check for improved blood flow after ascending aorta replacement.

\section{Disclosures}

Conflicts of interest: The authors have no conflict of interest.

\section{References}

1. Brown JW, Ruzmetzov M, Minnich DJ, et al. Surgical management of scimitar syndrome: An alternative approach. J Thorac Cardiovasc Surg 2003; 125: 238-45.

2. Cambria RP, Brewster DC, Gertler J, et al. Vascular complications associated with spontaneous aortic dissection. J Vasc Surg 1988; 7: 199-209.

3. Geirsson A, Szeto WY, Pochettino A, et al. Significance of malperfusion syndromes prior to contemporary surgical repair for acute type A dissection: outcomes and need for additional revascularizations. Eur J Cardiothorac Surg 2007; 32: 255-62.

4. Halasz NA, Halloran KH, Liebow AA. Bronchial and arterial anomalies with drainage of the right lung into the inferior vena cava. Circulation 1956; 12: 826-46.

5. Shiroma K, Ebine K, Tamura S, Yokomuro M, Suzuki H, Takanashi Y. A case of Turner's syndrome associated with partial anomalous pulmonary venous return complicated by dissecting aortic aneurysm and aortic regurgitation. J Cardiovasc Surg (Torino) 1997; 38: 257-9.

6. Chaikriangkrai K, Lopez-Mattei JC, Flores-Arredondo J, et al. Case Report: Magnetic Resonance Imaging of a Scimitar Vein and Aortic Dissection. Methodist Debakey Cardiovasc J 2014; 10: 257 . 